\title{
Nonmydriatic Ultra-Wide-Field Scanning Laser Ophthalmoscopy (Optomap) versus Two-Field Fundus Photography in Diabetic Retinopathy
}

\author{
Raffael Liegl $^{\mathrm{a}} \quad$ Kristine Liegl $^{\mathrm{b}} \quad$ Lala Ceklic $^{\mathrm{c}} \quad$ Christos Haritoglou $^{\mathrm{a}}$ \\ Anselm Kampik ${ }^{a}$ Michael W. Ulbig ${ }^{a}$ Marcus Kernt ${ }^{a} \quad$ Aljoscha S. Neubauer $^{a}$ \\ ${ }^{a}$ Department of Ophthalmology, Ludwig-Maximilians University Munich, Munich, and ${ }^{\mathrm{b}}$ Department of \\ Ophthalmology, Friedrich-Alexander University Erlangen-Nuremberg, Erlangen, Germany; ' Department of \\ Ophthalmology, 'Kasindo' Clinical Center of Eastern Sarajevo, East Sarajevo, Bosnia and Herzegovina
}

\begin{abstract}
The purpose of this study was to investigate the diagnostic properties of a 2-laser wavelength nonmydriatic $200^{\circ}$ ultrawide-field scanning laser ophthalmoscope (SLO) versus mydriatic 2-field $45^{\circ}$ color fundus photography (EURODIAB standard) for assessing diabetic retinopathy (DR). A total of 143 consecutive eyes of patients with different levels of DR were graded regarding DR level and macular edema based on 2-field color photographs or 1 Optomap Panoramic 200 SLO image. All SLO images were nonmydriatic and all photographs mydriatic. Grading was performed masked to patient and clinical data. Based on photography, 20 eyes had no DR, 44 had mild, 18 moderate and 42 severe nonproliferative DR, and 19 eyes had proliferative DR. Overall correlation for grading DR level compared to Optomap SLO was moderate with kappa 0.54 ( $p<0.001$ ), fair-to-moderate in macular edema grading with kappa 0.39 ( $p<0.001)$, and substantial for grading clinically significant macular edema (kappa 0.77). The wide-field SLO offers a wider field of view and can potentially better differentiate lesions by applying the 2 laser wavelengths. However, these advantages over 2-field fundus photography need to be confirmed in further studies.

(c) 2013 S. Karger AG, Basel
\end{abstract}

\section{Introduction}

Diabetic retinopathy (DR) and particularly diabetic macular edema (DME), microvascular complications of diabetes mellitus, are leading causes of visual impairment in developed countries. According to the World Health Organization (WHO) almost $2 \%$ of the world population is blind due to DR, and $10 \%$ are visually disabled [1]. Taking the rise from 170 million people affected by diabetes mellitus in 2000 to an estimated 366 million in 2030 into account, the absolute number of patients suffering from vision deterioration due to DR complications will increase significantly [2]. After 20 years all people with insulin-dependent diabetes mellitus and $60 \%$ with insulinnondependent diabetes mellitus have DR [3].

DME is the most common cause for vision decrease and the presence of DME increases the risk for this by 30 $50 \%[4,5]$. With the introduction of VEGF-targeted intravitreal therapeutic approaches, an even earlier detection of the DR grade including the presence of DME is needed.

Over the last decades many efforts to develop appropriate screening tools and protocols have been made. The rationale for the development of nonmydriatic screening

\section{KARGER}

E-Mail karger@karger.com www.karger.com/oph (c) 2013 S. Karger AG, Basel

0030-3755/13/2311-0031\$38.00/0
Raffael Liegl, MD

Department of Ophthalmology, Ludwig-Maximilians University Munich Mathildenstrasse 8

DE-80336 Munich (Germany)

E-Mail raffael.liegl@med.uni-muenchen.de 
techniques based on fundus photography and telemedicine programs included patient's comfort and needs (particularly in many screening programs in developed countries), as well as user-friendly application and handling in rural areas (where there is less availability of an ophthalmological expert) and the need for teleophthalmological networks.

The recognized 'gold standard' in obtaining retinal photographs is the Early Treatment Diabetic Retinopathy Study (ETDRS) standard 7-field $30^{\circ}$ color fundus photography [6]. However, although ETDRS photography and grading provide a reliable and proven method of documentation and assessment of DR-related fundus alterations, it is a time-consuming examination requiring skilled photographers and pharmacological pupil dilation. The complexity of this single examination might be one of the reasons why only an estimated $60 \%$ of the diabetic population in the USA receives recommended annual eye screenings [7].

In order to make this procedure more patient and user friendly several alterations in obtaining fundus photographs, including nonmydriatic cameras, digital video imaging and fewer-field photography, have been evaluated [8-10]. Among these, particularly 2 -field $45^{\circ}$ retinal photographs (1 macular-centered field and 1 disc/nasal field), are a well-established and validated screening standard as used and proposed in the EURODIAB IDDM complications study [11].

Ultra-wide-field scanning laser ophthalmoscopy (SLO) is a novel nonmydriatic fundus imaging device (Optomap Panoramic 200; Optos plc., Dunfermline, Scotland, UK) which allows imaging not only of the posterior pole of the retina in undilated pupils, but even extending over the equator. It covers $180-200^{\circ}$ with no need for pupil dilation, which has theoretical advantages over standard fundus photography. Moreover, it is well known that the SLO technique is less susceptible to media opacities (especially cataracts) [12] and to a decrease in pupil diameter. On the other hand, SLO optical resolution is limited and has numerically less pixels than fundus photography - but it yields a higher contrast [13]. We have previously shown that the Optomap SLO performance in assessing DR can compete with clinical examination [13]. However, assessment by photographic reading center grading is known to be superior to clinical examination $[14,15]$. In this patient series, we therefore sought to assess the value of wide-field SLO in DR screening application versus photography. This study compares the diagnostic properties of nonmydriatic $200^{\circ}$ ultra-wide-field SLO (Optomap) versus a photographic DR screening standard of two $45^{\circ}$ mydriatic fundus photographs.
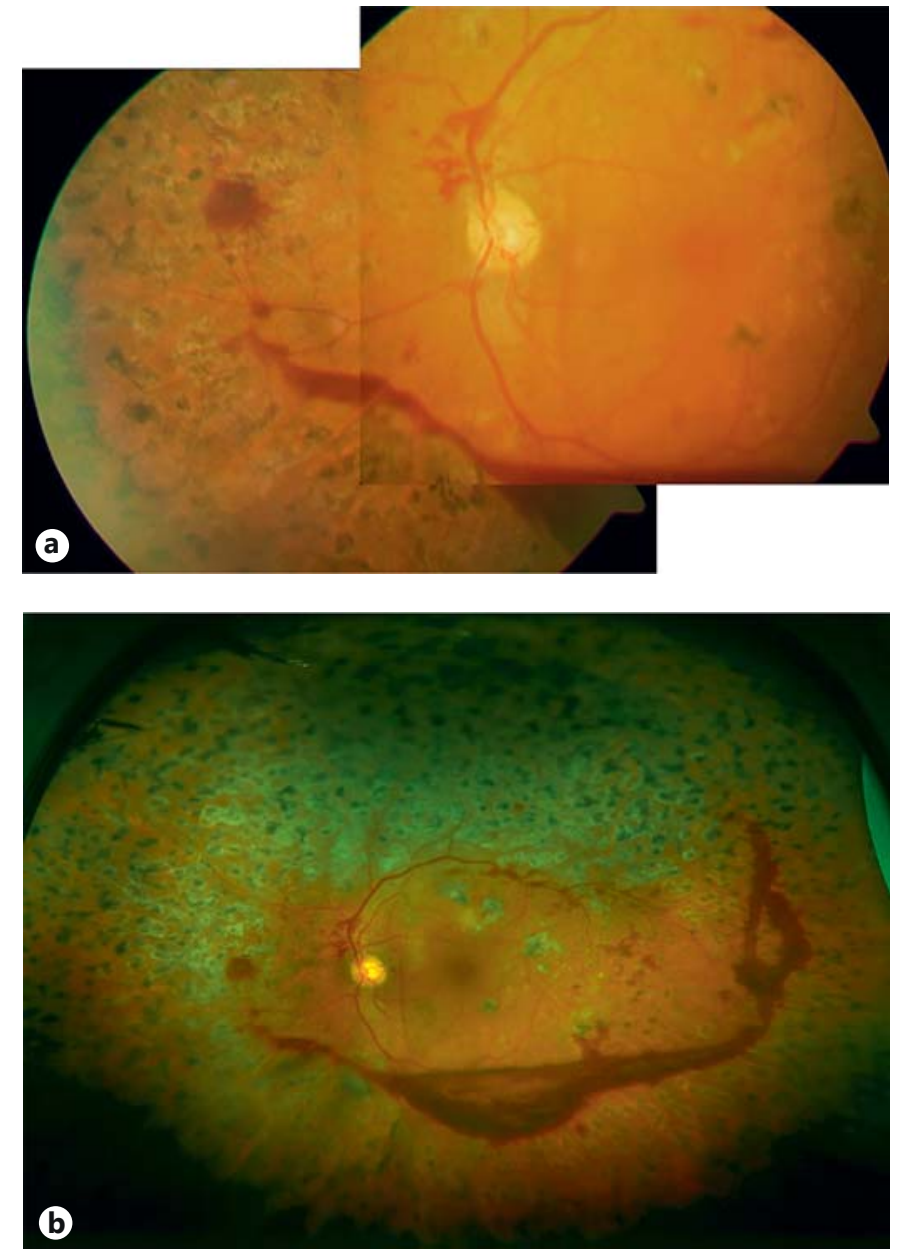

Fig. 1. Example of 2-field photography (a) and corresponding wide-field SLO imaging (b) of a patient. Despite panretinal laser coagulation a preretinal hemorrhage can be seen at the lower temporal vessel arcade with significant neovascularization. On widefield SLO imaging some lashes (lower part of the images) and parts from imaging optics (left and right periphery) are present.

\section{Methods}

Patients

Consecutive patients were recruited from the outpatient clinic of the Department of Ophthalmology, Ludwig-Maximilians-University, Munich. Patients were included if they had diabetes (based on WHO criteria) for at least 3 years. Eyes were excluded if there were eye diseases involving the posterior pole other than DR (such as age-related macular degeneration), but not for media opacities. Graders did not participate in clinical examination of the patients and had no access to clinical data.

\section{Optomap Imaging}

After informed consent, Optomap imaging was performed without pupil dilation before, and independently of, the clinical examination. The study conformed to the principles expressed in 
Table 1. Agreement in DR grading based on EURODIAB photography versus Optomap fundus photographs

\begin{tabular}{|c|c|c|c|c|c|c|}
\hline \multirow[t]{2}{*}{ Optomap grading } & \multicolumn{5}{|c|}{$\begin{array}{l}\text { Photography (EURODIAB) } \\
\text { classification of DR }\end{array}$} & \multirow[t]{2}{*}{ Total } \\
\hline & $\begin{array}{l}\text { no } \\
\text { DR }\end{array}$ & $\begin{array}{l}\text { mild } \\
\text { NPDR }\end{array}$ & $\begin{array}{l}\text { moderate } \\
\text { NPDR }\end{array}$ & $\begin{array}{l}\text { severe } \\
\text { NPDR }\end{array}$ & PDR & \\
\hline No DR & 17 & 5 & 0 & 0 & $1^{1}$ & 23 \\
\hline Mild NPDR & 3 & 26 & 2 & 0 & $1^{1}$ & 32 \\
\hline Moderate NPDR & 0 & 8 & 11 & 9 & 0 & 28 \\
\hline Severe NPDR & 0 & 5 & 4 & 27 & 6 & 42 \\
\hline PDR & 0 & 0 & 1 & 6 & 11 & 18 \\
\hline Total & 20 & 44 & 18 & 42 & 19 & 143 \\
\hline
\end{tabular}

EURODIAB photography-based grading of DR vs. assessment from Optomap images. The numbers give the number of eyes in each category. Overall correlation for DR level grading compared to Optomap was moderate with kappa of 0.54 ( $p<0.001)$.

${ }^{1}$ Both cases were verified to have no active PDR in regrading and in clinical examination: 1 patient had a fibrosed neovascularization of the disc - while the fibrosis and panretinal laser photocoagulation was not imaged in 2-field photography. Figure 2 shows the corresponding images. The other patient had no DR but due to low imaging quality in photography a disc neovascularization was suspected.

the Declaration of Helsinki and approval was obtained from the Institutional Review Board. Optomap imaging consisted of taking several images, where the best image per eye was saved on the server for grading. The instrument takes 1 image in approximately $0.25 \mathrm{~s}$, thus avoiding motion artifacts. Total scanning time approximates $3-5$ min including patient positioning and was performed by one of the authors (K.L.). Basic operation of the Optomap Panoramic 200 (an SLO) involves scanning with 2 laser wavelengths: 1 green $(532 \mathrm{~nm})$ and 1 red $(633 \mathrm{~nm})$ laser. The 2 images are then either viewed separately or superimposed by the software to yield semirealistic color imaging (see fig. 1). The instrument requires a small optical path of only $2 \mathrm{~mm}$ and by a special mirror design is able to obtain wide-field images of approximately $180-200^{\circ}$ without pupil dilatation. The optical resolution with the instrument used in this study was $3,900 \times 3,072$ pixels for that angle, resulting in approximately 15-21 pixels per degree. Due to the SLO principle, images of high contrast and sharpness were obtained [12].

\section{Two-Field $45^{\circ}$ Fundus Photography}

Prior to retinal photography, the patient's pupils were dilated using tropicamide $1 \%$ and additional epinephrine $10 \%$ if required - repeated if pupils did not reach at least $6 \mathrm{~mm}$ in diameter. Color retinal photographs, with a suitable high-quality retinal digital camera (Zeiss FF450; Carl Zeiss Meditec AG, Jena, Germany) were taken by a certified ophthalmic photographer. A 5.0-megapixel charge-coupled device sensor (Sony 3CCD; Sony, Tokyo, Japan) was utilized in this study. Focusing and alignment of the image were performed using the ocular tube of the camera in addition to a previewing camera. The two $45^{\circ}$ fields, according to EURO-
DIAB [11], were obtained as follows: (1) macular field: positioned such that the exact center of the optic disc lay at the nasal end of the horizontal meridian of the field of view and (2) disc/nasal field: such that the optic disc was positioned 1 disc diameter in from the temporal edge of the field, on the horizontal meridian.

\section{Grading of Images}

All retina images were loaded from the server to a viewing station (equipped with a conventional cathode ray 17-inch noncalibrated color monitor) via network and assessed with the Optomap viewing software (Optomap U-revu, version 1.0). This software allows basic image manipulations such as changing contrast and brightness and zooming. It also offers viewing both in the composite color image and the single-color wavelengths. The images obtained by the different wavelengths were utilized to better identify and differentiate lesions (especially red-free image [16]). The grader (L.C.) had not participated in the examination of the patients and was masked to all additional information such as visual acuity, duration of diabetes or clinical symptoms. The grader, however, could decide not to grade due to insufficient image quality, which was defined as not covering at least the central $60^{\circ}$ and both the macula and the optic disc in adequate quality. Nongradable images were reassessed by a second grader (A.S.N.) to reach consensus on gradability. The level of DR and macular edema were assessed using the International Clinical Diabetic Retinopathy (ICDR) Severity Scale [17]. Independently of this assessment the presence of clinically significant macular edema (CSME) was graded according to ETDRS classification [18].

\section{Statistics}

All data were collected in an MS-Excel 2000 spreadsheet (Microsoft Corporation, Redmond, Wash., USA) and analyzed using SPSS 17.0 for Windows (SPSS Inc., Chicago, Ill., USA). In all tests $\mathrm{p}<0.05$ was considered significant. Kappa statistics were calculated and assessed as proposed by Altman [19]: $<0.20$ poor, $0.21-$ 0.40 fair, $0.41-0.60$ moderate, $0.61-0.80$ substantial and $0.81-1.00$ almost perfect strength of agreement. Unweighted kappa was used, thus avoiding any bias by weighting.

\section{Results}

A total of 143 eyes, 74 of which were left eyes, of 74 patients were included in the study. Mean $( \pm$ SD) patient age was $60 \pm 12.1$ years (range $24-75$ years). Diabetes duration ranged from 1 to 31 years (mean $13.4 \pm 10.1$ years). Of all patients, $53 \%$ were using insulin while $47 \%$ were on oral medication. Mean $\mathrm{HbA}_{1 \mathrm{c}}$ was $7.51 \pm 1.46 \%$ (range 8-10\%).

Grading of DR based on the international classification scale is presented in table 1 . According to the $45^{\circ}$ photos, 20 (13.9\%) eyes had no DR, 44 (30.8\%) had mild, 18 (12.6\%) moderate and $42(29.4 \%)$ severe nonproliferative DR (NPDR), and 19 (13.2\%) eyes had proliferative DR (PDR). The strength of agreement between these 2 imaging methods was moderate with a kappa statistic of $0.54(\mathrm{p}<0.001)$. For the 2 cases not identified as PDR by 

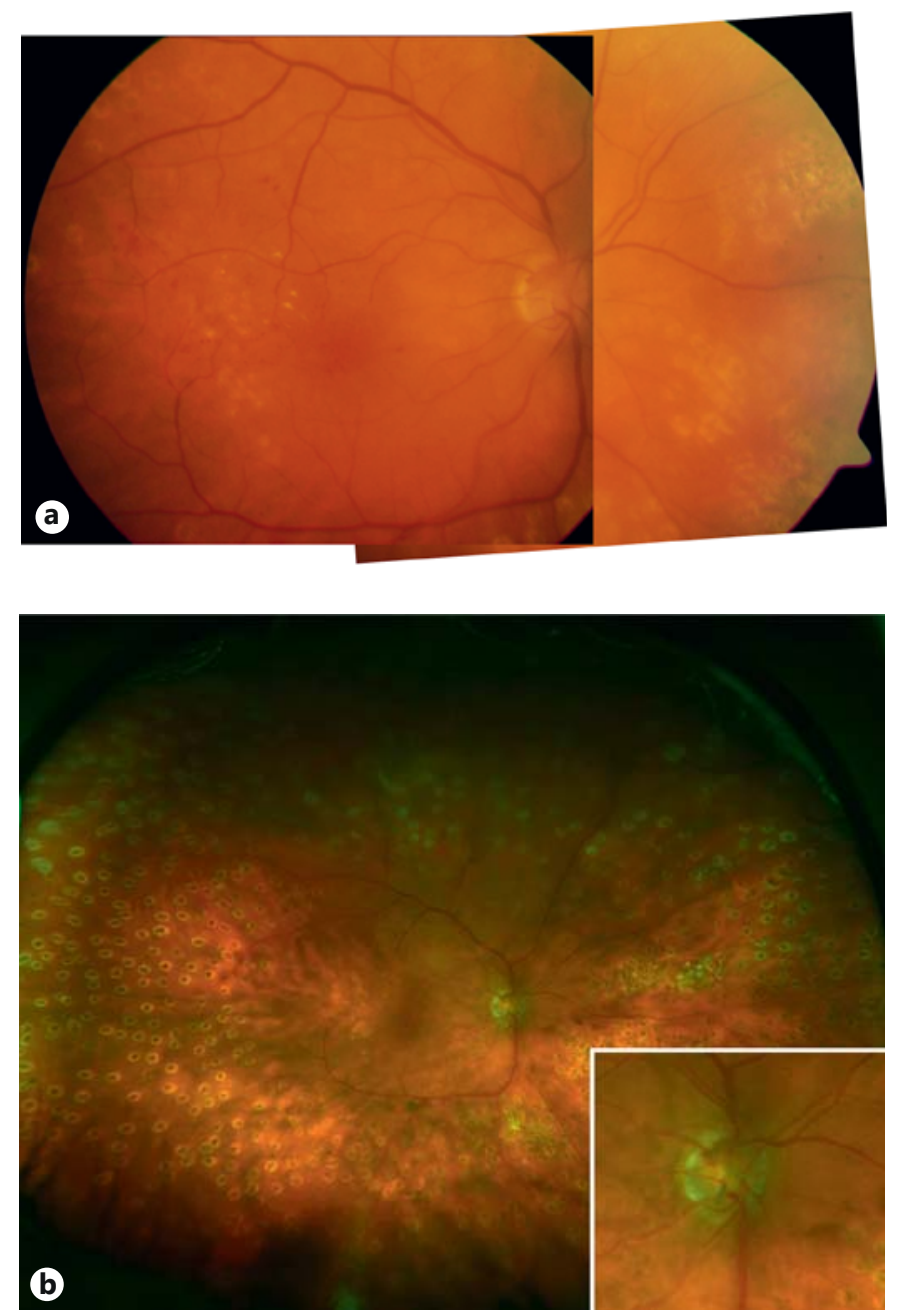

Fig. 2. Example of 2-field photography (a) and corresponding wide-field SLO imaging (b) of a patient with PDR and fibrotic neovascularization at the optic disc. The patient was falsely graded to be active PDR based on fundus photography (a), while SLO imaging clearly showed the fibrosis, especially when viewed at higher magnification as shown in the lower right inset (b).

Optomap imaging (table 1), these were confirmed clinically and on regrading were determined to be nonproliferative. Figure 2 illustrates 1 case.

Based on the international clinical classification EURODIAB photography yielded macular edema absent in 61 eyes (42.6\%), mild in 62 (43.3\%), moderate in $14(9.7 \%)$ and severe in 6 eyes ( $4.2 \%$; table 2 ). Concordance regarding macular edema grading was fair-to-moderate with a kappa value of $0.39(\mathrm{p}<0.001)$. Table 2 shows results and corresponding Optomap grading in detail. On photography, CSME was graded to be present in 38 eyes (26.6\%), while 105 (73.4\%) of the eyes did not have CSME. Table 3
Table 2. Concordance in grading severity of DME according to international classification based on EURODIAB photography versus Optomap

\begin{tabular}{lrllll}
\hline \multirow{2}{*}{$\begin{array}{l}\text { Optomap } \\
\text { grading }\end{array}$} & \multicolumn{5}{c}{ Photography (EURODIAB) classification of DME } \\
\cline { 2 - 6 } & no ME & $\begin{array}{l}\text { mild } \\
\text { ME }\end{array}$ & $\begin{array}{l}\text { moderate } \\
\text { ME }\end{array}$ & $\begin{array}{l}\text { severe } \\
\text { ME }\end{array}$ & Total \\
\hline No ME & 54 & 21 & 2 & 0 & 77 \\
Mild ME & 5 & 21 & 4 & 0 & 30 \\
Moderate ME & 1 & 16 & 7 & 3 & 27 \\
Severe ME & 1 & 4 & 1 & 3 & 9 \\
Total & 61 & 62 & 14 & 6 & 143 \\
\hline
\end{tabular}

The numbers give the number of eyes in each category. Concordance regarding DME grading was fair-to-moderate with a kappa of 0.39 ( $\mathrm{p}<0.001)$. ME = Macular edema.

Table 3. Concordance in grading CSME based on EURODIAB photography versus Optomap

\begin{tabular}{llcc}
\hline \multirow{2}{*}{$\begin{array}{l}\text { Optomap } \\
\text { grading }\end{array}$} & \multicolumn{3}{l}{ Photography (EURODIAB) assessment of CSME } \\
\cline { 2 - 4 } & CSME present & no CSME & Total \\
\hline CSME present & 18 & 9 & 27 \\
No CSME & 20 & 96 & 116 \\
Total & 38 & 105 & 143 \\
\hline
\end{tabular}

The numbers give the number of eyes graded in each category. Sensitivity: $48 \%$; specificity: $91 \%$.

shows corresponding Optomap results. Both imaging methods have substantial agreement in the detection and evaluation of CSME (kappa value 0.77 ).

\section{Discussion}

The current study demonstrates that nonmydriatic ultra-wide-field SLO (Optomap) correlates moderately with mydriatic 2 -field $45^{\circ}$ photography. This adds to previous data, which have shown validity of assessments compared to clinical fundus examination and a lower rate of nongradable images for Optomap [13]. In an environment that demands cost reduction, practice efficiency and effective disease screening, nonmydriatic digital fundus screening offers many advantages. One important advantage inherent to nonmydriatic digital 
techniques is patient comfort (no need for dilating pupils with inability to drive a car) and facilitation of remote diagnostic image generation and interpretation. These factors are likely to improve patient preference and compliance in screening programs [20]. Another benefit of the Optomap nonmydriatic camera is its use of the SLO technique, as the image quality in opaque media is significantly better compared to standard cameras and thicker cataracts are more often seen in low-income and rural areas [21]. Additionally, remote interpretation could permit screening of underserved and low-income rural and urban populations at risk for DR [15]. The Digital Diabetic Screening Group suggests that a nonmydriatic digital photography protocol is superior to mydriatic ophthalmoscopy and roughly comparable to 7 -field ETDRS mydriatic photography.

Most fundus camera-based screening systems for DR take one or two $45^{\circ}$ or $60^{\circ}$ images. This has been validated against the best known standard for fundus photography in DR, the 7-field ETDRS photography, which covers 75$65^{\circ}$ of the central retina by montaging seven $30^{\circ}$ photographs. The ETDRS standard is known to have good validity - given the fact that photographic grading was validated against long-term outcomes [22] - but is difficult to perform and relatively unsuitable for routine. While two $60^{\circ}$ fundus photographs ( 1 macula-centered and 1 optic disc-centered) cover $80 \%$ of this area and make it unlikely that areas of neovascularization will be missed [23], most screening programs today cover significantly less retinal area. These programs still offer good screening characteristics [24], although the relatively small covered area may theoretically reduce sensitivity. However, these standards appear to be sufficient for screening purposes as carried out elsewhere when the standard using two $45^{\circ}$ photographs, as in our study (EURODIAB protocol), was validated successfully against 7-field ETDRS photography, the diagnostic gold standard [11]. Without any doubt, a larger field of view offers better detection characteristics; however, coverage of the nasal retina appears to be more important than the field of view in total. In terms of area coverage, the ultra-wide-field SLO investigated here (see fig. 1) clearly exceeds the ETDRS standard, which could offer additional advantages. For instance, in 1 of the cases falsely classified as PDR based on photography (table 1), Optomap imaging quality and field of view could exclude proliferations.

The SLO technique showed only a fair-to-moderate agreement with photography regarding macular edema grading (tables 2,3). It should be noted though that nonstereoscopic fundus photography is equally limited re-

Wide-Field SLO vs. 2-Field Photography in DR garding detection of thickening as none of these imaging modalities create 3 -dimensional photographs. Therefore, although a discrepancy between Optomap and standard photography (EURODIAB) graded pictures exist (table 3), these results have to be considered with caution as standard photographs do not represent a common standard in diagnosing macula edema. The results are particularly surprising since a previous study comparing Optomap photographs to stereo biomicroscopy with three independent graders revealed a specificity of $100 \%$ and a sensitivity of $94 \%$ compared to only $48 \%$ specificity and $91 \%$ sensitivity in comparison to standard photography in this study [13] (table 3). Another study compared stereoscopic digital fundus photography against contact lens biomicroscopy for the detection of CSME with a specificity of around $50-90 \%$ and a sensitivity of $90-99 \%$, a result very comparable to ours when comparing Optomap against standard photography [25]. Taken together, there is no doubt that photographic assessment is suitable for detecting sight-threatening diseases and the course of these diseases (as in PDR), regardless of whether it is based on standard photography or SLO technique. Photography, however, cannot reliably detect 3-dimensional changes (as in macular edema). Therefore, grading of macular thickening always requires stereoscopic assessment by biomicroscopy and/or a cross-sectional technique such as OCT. Considering these different results in terms of the ability of photographic assessment for determining CSME, the real value of solely grading fundus images with regard to CSME by Optomap cannot be fully determined.

Limitations of the Optomap system have been described earlier and include misdiagnosing and missing lesions, which were not relevant in this study [26]. The percentage of nongradable images, which is a relevant marker in screening applications, cannot be assessed in this report. Only patients receiving both imaging technologies were included and in those no eyes had to be excluded for nongradable images. While this is a limitation of our study, in a similar patient series evaluated previously for Optomap nongradable and 'nonobtainable' images, the numbers were approximately $<10 \%$ of all patients [13].

In summary, the nonmydriatic ultra-wide-field SLO images are of sufficient quality to assess the DR level validly. Compared to a screening standard (EURODIAB) of two $45^{\circ}$ photographs, inter-method agreement was only moderate. The additional field of view from Optomap may offer additional advantages and yield other incidental fundus abnormalities.

Ophthalmologica 2014;231:31-36

DOI: $10.1159 / 000355092$ 


\section{Acknowledgments}

The authors thank Mrs. S. Guthmann for expert technical assistance with Optomap and Lisa Merz and Gabriele Maltzahn for expert fundus photography. Parts of this study were presented at the World Congress of Ophthalmology, 2010 in Berlin.

\section{Disclosure Statement}

The authors have no commercial interest in any of the materials and methods used in this study.

\section{References}

1 World Health Organization: Diabetes. What is diabetes? Fact sheet No 312, 2006. http:// www.who.int/mediacentre/factsheets/fs312/ en/ (accessed December 12, 2007).

2 Medina FJ, Callen CI, Rebolleda G, MunozNegrete FJ, Callen MJ, del Valle FG: Use of nonmydriatic spectral-domain optical coherence tomography for diagnosing diabetic macular edema. American J Ophthalmol 2012;153:536-543.

3 World Health Organization: Prevention of blindness and visual impairment. Diabetic retinopathy. 2009. http://www.who.int/ blindness/causes/priority/en/index6.html (accessed December 12, 2009).

4 Bresnick GH: Diabetic macular edema. A review. Ophthalmology 1986;93:989-997.

5 Virgili G, Menchini F, Dimastrogiovanni AF, Rapizzi E, Menchini U, Bandello F, Chiodini RG: Optical coherence tomography versus stereoscopic fundus photography or biomicroscopy for diagnosing diabetic macular edema: a systematic review. Invest Ophthalmol Vis Sci 2007;48:4963-4973.

6 Early Treatment Diabetic Retinopathy Study Research Group: Grading diabetic retinopathy from stereoscopic color fundus photographs - an extension of the modified Airlie House classification. ETDRS report number 10. Ophthalmology 1991;98:786-806.

7 Schoenfeld ER, Greene JM, Wu SY, O'Leary E, Forte F, Leske MC: Recruiting participants for community-based research: the diabetic retinopathy awareness program. Ann Epidemiol 2000;10:432-440.

8 Penman AD, Saaddine JB, Hegazy M, Sous ES, Ali MA, Brechner RJ, Herman WH, Engelgau MM, Klein R: Screening for diabetic retinopathy: the utility of nonmydriatic retinal photography in Egyptian adults. Diabet Med 1998;15:783-787.

9 Fransen SR, Leonard-Martin TC, Feuer WJ, Hildebrand PL: Clinical evaluation of patients with diabetic retinopathy: accuracy of the Inoveon diabetic retinopathy-3DT system. Ophthalmology 2002;109:595-601.
10 Bresnick GH, Mukamel DB, Dickinson JC, Cole DR: A screening approach to the surveillance of patients with diabetes for the presence of vision-threatening retinopathy. Ophthalmology 2000;107:19-24.

11 Aldington SJ, Kohner EM, Meuer S, Klein R, Sjolie AK: Methodology for retinal photography and assessment of diabetic retinopathy: the EURODIAB IDDM complications study. Diabetologia 1995;38:437-444.

12 Kirkpatrick JN, Manivannan A, Gupta AK, Hipwell J, Forrester JV, Sharp PF: Fundus imaging in patients with cataract: role for a variable wavelength scanning laser ophthalmoscope. Br J Ophthalmol 1995;79:892-899.

13 Neubauer AS, Kernt M, Haritoglou C, Priglinger SG, Kampik A, Ulbig MW: Nonmydriatic screening for diabetic retinopathy by ultra-widefield scanning laser ophthalmoscopy (Optomap). Graefes Arch Clin Exp Ophthalmol 2008;246:229-235.

14 Whited JD, Datta SK, Aiello LM, Aiello LP, Cavallerano JD, Conlin PR, Horton MB, Vigersky RA, Poropatich RK, Challa P, Darkins AW, Bursell SE: A modeled economic analysis of a digital tele-ophthalmology system as used by three federal health care agencies for detecting proliferative diabetic retinopathy. Telemed J E Health 2005;11:641651.

15 Lin DY, Blumenkranz MS, Brothers RJ, Grosvenor DM: The sensitivity and specificity of single-field nonmydriatic monochromatic digital fundus photography with remote image interpretation for diabetic retinopathy screening: a comparison with ophthalmoscopy and standardized mydriatic color photography. Am J Ophthalmol 2002;134:204-213.

16 Lin DY, Blumenkranz MS, Brothers R: The role of digital fundus photography in diabetic retinopathy screening. Digital Diabetic Screening Group (DDSG). Diabetes Technol Ther 1999;1:477-487.
17 Wilkinson CP, Ferris FL 3rd, Klein RE, Lee PP, Agardh CD, Davis M, Dills D, Kampik A, Pararajasegaram R, Verdaguer JT: Proposed international clinical diabetic retinopathy and diabetic macular edema disease severity scales. Ophthalmology 2003;110:1677-1682.

18 Early Treatment Diabetic Retinopathy Study Research Group: Photocoagulation for diabetic macular edema. ETDRS report number 1. Arch Ophthalmol 1985;103:1796-1806.

19 Altman DG: Practical statistics for medical research. London, Chapman \& Hall, 1991.

20 Cavallerano AA, Cavallerano JD, Katalinic P, Blake B, Rynne M, Conlin PR, Hock K, Tolson AM, Aiello LP, Aiello LM: A telemedicine program for diabetic retinopathy in a Veterans Affairs Medical Center - the Joslin Vision Network Eye Health Care Model. Am J Ophthalmol 2005; 139:597-604.

21 Beckman C, Bond-Taylor L, Lindblom B, Sjostrand J: Confocal fundus imaging with a scanning laser ophthalmoscope in eyes with cataract. Br J Ophthalmol 1995;79:900-904.

22 Early Treatment Diabetic Retinopathy Study Research Group: Fundus photographic risk factors for progression of diabetic retinopathy. ETDRS report number 12. Ophthalmology 1991;98:823-833.

23 von Wendt G, Ronnholm P, Heikkila K, Summanen P: A comparison between one- and two-field $60^{\circ}$ fundus photography when screening for diabetic retinopathy. Acta Ophthalmol Scand 2000;78:14-20.

24 Stellingwerf C, Hardus PL, Hooymans JM: Two-field photography can identify patients with vision-threatening diabetic retinopathy: a screening approach in the primary care setting. Diabetes Care 2001;24:2086-2090.

25 Rudnisky CJ, Hinz BJ, Tennant MT, de Leon AR, Greve MD: High-resolution stereoscopic digital fundus photography versus contact lens biomicroscopy for the detection of clinically significant macular edema. Ophthalmology 2002;109:267-274.

26 Chou B: Limitations of the Panoramic 200 Optomap. Optom Vis Sci 2003;80:671-672. 